\title{
A 10 Year Review of 255 cases of Ectopic Pregnancy
}

\begin{abstract}
Keywords: Ectopic Pregnancy ; Pelvic Inflammatory Disease Culdocentesis

Abstract

Objective: The study was conducted to determine the frequency, risk factors, clinical features and diagnostic modalities of Ectopic Pregnancy in a tertiary care hospital.

Study design: Retrospective, Analytic study.

Place and duration of study: Gynae B ward, Ayub Teaching hospital, Abbottabad, from Jan 2003 to Dec 2012.

Methodology: The patients included in this study were admitted from outpatient department and emergency department to gynaecological unit. A detailed history was taken and examination was done. Variables like age of the patient, parity, past history, symptoms, signs, and operative procedure were all recorded for evaluation. Various predisposing factors like previous abortion, contraception, recurrent ectopic, previous pelvic surgery, infertility treatment and pelvic inflammatory disease were analyzed.

Results: There were 255 cases of Ectopic pregnancy out of 25010 deliveries during the study period and the frequency was $1.01 \%$. The main predisposing factor to ectopic pregnancy was pelvic inflammatory disease (43.13\%) while $37.64 \%$ had previous abortions. Recurrent ectopic pregnancy was observed in $9.01 \%$ of patients while $27.05 \%$ had history of previous pelvic surgeries.
\end{abstract}

Conclusion: The most common risk factor for ectopic pregnancy is pelvic inflammatory disease. If it is timely diagnosed and properly treated, ectopic pregnancy with its grave consequences can be avoided.

\section{Introduction}

Implantation of a pregnancy outside the uterine cavity was first described in England by Gifford in 1731 [1]. Its incidence has increased from 0.5 per hundred pregnancies thirty years ago to the present day quoted incidence of 2 per hundred pregnancies [2,3]. The incidence of ectopic pregnancy in Pakistan varies from 1:112 to 1:130 pregnancies. $[4,5]$. In African women the risk of ectopic pregnancy is 1.6 times greater than the risk amongst whites (13 per 1000) [6]. The reason for this increase is probably high incidence of pelvic inflammatory disease and the use of intra-Uterine contraceptive device [7]. Several risk factors for ectopic pregnancy have been identified including a history of pelvic inflammatory disease, smoking, previous ectopic pregnancy, pelvic surgery, ovulation induction and the use of intrauterine contraceptive device [8]. Ectopic pregnancy remains an important cause of maternal mortality worldwide. In the UK both the mortality rate at 4 per 10,000 pregnancies [9]. In order to decrease maternal mortality and morbidity due to ectopic pregnancy, there is a need for early diagnosis. With the advent of transvaginal scan and sensitive serum BHCG earlier diagnosis of ectopic pregnancy can be made. The aim of this study was to review the data of ectopic pregnancies during the last 10 years. Risk factors contributing to ectopic pregnancy, clinical features and diagnostic modalities were also evaluated.
Journal of

\section{Andrology \& Gynaecology}

\author{
Bushra Khan ${ }^{1 *}$, Farhat Deeba ${ }^{2}$ and Wajiha Khan ${ }^{3}$ \\ ${ }^{1}$ Gynae B unit, Ayub Teaching hospital, Abbottabad, Pakistan \\ ${ }^{2}$ Women Medical College, Abbottabad, Pakistan \\ ${ }^{3}$ COMSATS institute of Information technology, Department of \\ Enviromental Sciences, Abbottabad, Pakistan \\ Address for Correspondence \\ Bushra Khan, Senior Registrar, Gynae B unit, Ayub Teaching hospital, \\ Abbottabad Pakistan, E-mail: alrazimedicalstore@yahoo.com \\ Submission: 26 September 2013 \\ Accepted: 25 November 2013 \\ Published: 27 November 2013
}

\section{Methodology}

The study was conducted in Ayub Teaching Hospital, Abbottabad in the department of Gynae \& obstetrics from January 2003 to December 2012. The patients included in this study were from outpatient department and emergency department. Patients who had any four of the following features are included in the study, i.e history of amenorrhea not more than 12 weeks, positive pregnancy test, abdominal pain ,empty uterus on scan, adenexal mass, positive culdocentesis.

A detailed history regarding age of the patient, parity, symptoms like amenorrhoea, per vaginal bleeding, abdominal pains, dizziness and vomiting was taken. Past history of repeated abortions, surgical interventions, infertility, pelvic inflammatory disease, history of contraception and ovulation induction was also taken. Pelvic inflammatory disease was diagnosed on the basis of history of repeated foul smelling vaginal discharge, fever and repeated antibiotic cover. Patients were particulary asked about previous record of ultrasound to see whether they had intrauterine or extrauterine pregnancy.

Patients were examined and signs like shock, features of peritonism, abdominal tenderness, cervical excitation and adnexal tenderness were noted. Paracentesis and culdocentesis were also performed in some cases. Provisional diagnosis of ectopic pregnancy was made either by history and clinical examination alone or was supplemented by investigations like trans abdominal scan, trans vaginal scan, urinary beta HCG and serum beta HCG. Diagnostic laparoscopy was not considered in any case. Patients who came in shock were examined in detail and based on history and examination, diagnosis of rupture ectopic was made. After initial stabilization and arrangement of blood laparotomy was carried out preceded by informed consent.

In some cases surgical opinion was also taken as the symptoms mimicked acute appendicitis. Most of the patients with ruptured ectopic pregnancy had free fluid in the pouch of Douglas on ultrasonography. Some of our patients who were diagnosed as cases of chronic ectopic and unruptured ectopic gestations by ultrasound scan done in private hospitals were included in the study and investigations like urinary beta HCG, serum beta HCG and trans vaginal scan were carried out to confirm diagnosis. 
Patients presented with acute abdomen who had ultrasound features of ovarian cyst with empty uterus but raised beta HCG were also included in the study.

\section{Results}

There were 255 cases of ectopic pregnancy out of 25010 deliveries during the study period, making it $1.01 \%$ of the total deliveries. Age ranged from 15 to 42 years. Majority of the patients, $222(82.73 \%)$ were in the age group of $21-30$ years (Table 1). Sixty three (20.4\%) were primigravidae, $140(54.9 \%)$ were multigravidae and $52(20.4 \%)$ were grandmultigravidae.

Risk factors for ectopic pregnancy were evaluated (Table 2). In our study, One hundred and ten patients (43.13\%) had pelvic inflammatory disease, 96 (37.64\%) had previous abortions, 54 patients were using various methods of contraception, out of these, $27(10.58 \%)$ had history of IUCD use. Recurrent ectopic pregnancy was observed in $23(9.01 \%)$ patients. Sixty nine patients $(27.05 \%)$ had history of previous pelvic surgeries while infertility treatment mostly ovulation induction was taken by 45 patients (17.64\%).

The most common symptom at presentation was amenorrhea of greater than four weeks. There were 250 (98.03\%) patients who had amenorrhea at the time of presentation. This was followed by abdominal pain which was observed in 245 (96.07\%) patients. Two hundred and five (80.39\%) patients had per vaginal bleeding. Dizziness and vomiting were observed in 138 (54.11\%) and 78 (30.58\%) patients respectively. Majority, 189 (74.11\%) presented in shock with ruptured ectopic pregnancy and very few patients, 5 (1.96\%) were asymptomatic.

The most common sign observed in these patients was abdominal tenderness. There were 237 (92.94\%) patients who had tender abdomen at the time of presentation. Seventy four (29.01\%) patients

Table 1: Age of patients.

\begin{tabular}{|l|c|c|}
\hline Age(years) & Number of patients & Percentage \\
\hline $15-20$ & 15 & $5.88 \%$ \\
\hline $21-25$ & 111 & $43.52 \%$ \\
\hline $26-30$ & 100 & $39.21 \%$ \\
\hline $31-35$ & 22 & $8.62 \%$ \\
\hline $36-40$ & 5 & $1.96 \%$ \\
\hline $41-42$ & 2 & $0.78 \%$ \\
\hline Total & 255 & $100 \%$ \\
\hline
\end{tabular}

Table 2: Risk Factors of Ectopic Pregnancy.

\begin{tabular}{|l|l|c|c|}
\hline S.No & Risk factors & No & Percentage \\
\hline 01 & PID & 110 & $43.13 \%$ \\
\hline 02 & Previous Abortion & 96 & $37.64 \%$ \\
\hline 03 & Previous Pelvic Surgery & 69 & $27.05 \%$ \\
\hline 04 & Contraception & 54 & $21.17 \%$ \\
\hline 05 & Infertility Treatment & 45 & $17.64 \%$ \\
\hline 06 & Recurrent Ectopic pregnancy & 23 & $9.01 \%$ \\
\hline 07 & No Risk Factor & 39 & $15.29 \%$ \\
\hline
\end{tabular}

had adnexal mass while 163 (63.92\%) had cervical excitation. There were two hundred and five patients (80.39\%) who had fullness in pouch of Douglas.

Diagnostic procedures which were carried out included culdocentesis, paracentesis, trans abdominal scan, transvaginal scan, urinary BHCG and serum BHCG. Culdocentesis was carried out in 160 patients and was positive in 140 cases (87.5\%). Paracentesis was carried out in 73 patients and was positive in 70 patients (95.8\%). Transabdominal scan was positive in $79.3 \%$ while transvaginal was positive in $93.5 \%$ patients. It was seen that serum BHCG was positive in $90.44 \%$ while urinary BHCG was positive in $70.7 \%$.

In most of the cases salpingectomy was the treatment which was carried out. It was observed that in 231 cases salpingectomy was carried out while in 11 cases salpingotomy was done. Medical treatment was carried out only in 5 cases while 8 cases were treated conservatively.

\section{Discussion}

The incidence of ectopic pregnancy in our study was found to be 10.1 per 1000 deliveries. In recent years, the incidence of ectopic pregnancy has not varied remarkably [10-12]. Ectopic pregnancy is common in the reproductive age. The most common age group in this study was found to be between 21-30 years and majority of them were multigravidae. These results are comparable with other studies $[14,15,16]$. However, Manjhi et al. [17] showed increased risk of ectopic pregnancy amongst primigravidae which is conflicting with the results of our study. Our findings are more appropriate in ectopic pregnancy compared to the later study probably due to the fact that repeated pregnancies leads to greater wear and tear of uterus as a result inflammatory response may involve fallopian tubes as well.

The exact etiology of ectopic pregnancy is not known but different risk factors have been implicated as contributing factors [7]. In our study 216 patients $(84.70 \%)$ had risk factors associated with ectopic pregnancy. Majority of them had two or more risk factors. This finding was however higher than $33 \%$ and $60.5 \%$ reported by Khaleequee [15] and Shah [18] respectively. One hundred and ten patients (43.13\%) had pelvic inflammatory disease in our study. A previous study conducted by Lund, in Sweden from 1960 to 1975 provided a very strong etiologic evidence of association of pelvic inflammatory disease with ectopic pregnancy [19]. Intrauterine contraceptive device prevents implantation of ovum in the uterine cavity but leaves the other sites unprotected. In our study 54 patients used various methods of contraception. Out of these, $24 \%$ were using intra uterine contraceptive device. Intra uterine contraceptive device usage was also found to be associated with ectopic pregnancy in a study conducted by shaista [20] in the Kingdom of Saudia Arabia and Bouyer [21] in France. Previous abortions increase the risk of ectopic pregnancy [22]. In our study there were 96 (37.6\%) patients who had previous abortions, this was comparable to the findings by Naila and co-author [16]. The higher risk could be the consequence of uterine injuries consecutive to this procedure, either inflammatory lesions or asymptomatic ascending infections, in other words, due to injuries, inflammations, and infections resulting from the scraping and suctioning of a woman's uterus in a surgical abortion. These inflammations or lesions may damage the fallopian tube, inhibiting the transport of the embryo. Instead of implanting in the uterine wall, the embryo implants in the fallopian tube [23]. Studies have shown that the association of ectopic pregnancy with previous pelvic surgery 
may be due to peritoneal and peritubal adhesions that occur following these surgeries $[22,24]$.

The most common clinical presentation of ectopic pregnancy was amenorrhea and pain abdomen followed by vaginal bleeding, dizziness and vomiting which were consistent with findings of other studies $[25,26]$. In our study $74.11 \%$ of all women presented with shock at admission. This finding was also consistent with that of a study conducted in Nigeria [26] which showed a higher proportion of patients presenting with shock as compared to some series showing fewer women presenting with shock $[27,28]$.

In a study conducted in Guinea [29], amenorrhea and abdominopelvic pain were the main symptoms and closely resembled our study. Recently Shrestha and her colleague [30] in their study conducted in Kathmandu reported abdominal pain as the leading symptom and occurred in $100 \%$ of ectopic pregnancy .

Diagnosis of ectopic pregnancy was made either by clinical features alone or by using methods like culdocentesis, paracentesis, transabdominal scan, trans vaginal scan, serum BHCG and urinary BHCG. In our study clinical features were used alone to make a diagnosis of ectopic pregnancy in most of the cases of ruptured ectopic pregnancy. Culdocentesis was found to be a useful diagnostic procedure in $87 \%$ cases while paracentesis in $95 \%$ cases. All patients were not subjected to culdocentesis or para centesis. Culdocentesis is less commonly performed in current settings because transvaginal scan and quantitative BHCG measurement have better sensitivity. Culdocentesis remains an important diagnostic tool for ectopic pregnancy in a country like Pakistan where financial constrains and lack of availability of modern method is still prevailing (scan, serum BHCG). More over most of the cases reaching hospital are with ruptured ectopic, where culdocentesis is mostely positive. Culdocentesis was found to be a useful diagnostic procedure in various others studies as well $[16,31,32]$.

In present study serum BHCG (90\%) was found to be more effective in diagnosing ectopic pregnancy as compared to urinary GHCG (70\%). The results are comparable to reports from a study conducted by Naila [16] however, are different from findings of Majhi [17].

In our study $95 \%$ patients had surgical treatment of ectopic pregnancy while only $5 \%$ had either medical or conservative treatment.The reason for this high rate of surgical intervention was that most of the cases encountered were with ruptured ectopic and more over facilities for medical or conservative treatment were not easily available. Pakistan is a developing country. Health awareness programs need to be started. Latest diagnostic modalities should be available so that patients should present earlier with intact ectopic rather than ruptured ectopic. In various studies $[16,25]$ conducted in different parts of the world surgical treatment remained an important method of treatment.

\section{Conclusion}

Pelvic inflammatory disease and previous abortions were the important predisposing factors. Most of the patients presented with ruptured ectopic and surgical management remained an important method of treatment.

\section{References}

1. Jurkovic D (2012) Ectopic pregnancy. In: Edmonds DK, eds. Dewhurst's Textbook of obstertrics and gynaecology, $8^{\text {th }}$ edition. Blackwell Science: 76 .

2. Hankis GD, Clark SL, Cunningham FG, Gilstrap LC (1995) Ectopic Pregnancy. In: Dilmond E; Gilstrap. Operative obstetrics. New York: Appleton and Lange: 437-456.

3. Lehner R, Kucera E, Jirecek S, Egarter C, Husslein P (2000) Ectopic pregnancy. Arch Gynecol Obstet 263: 87-92.

4. Mazhar SB, Mahmood G, Parveen F (1998) Systemic methotrexate for the treatment of ectopic pregnancy larger than $3.5 \mathrm{cms}$. The $\mathrm{XVI}$ th Asian and Oceanic Congress of Obstetrics and Gynaecology. June $14-19^{\text {th }}$, Kuala Lumpur, Malaysia; 17-20.

5. Khawaja NP, Rehman R, Durrani Z (1998) Ectopic pregnancy at gynecology unit II Sir Ganga Ram Hospital ,Lahore; study of fifty cases. Pak J Obstet Gynecol 11: 61-65.

6. Konje JC, Potdar N (2011) Ectopic pregnancy. In: Shaw RW, Luesley D Monga A.eds.Gynaecology, 4th edition. Churchill Livingstone 363.

7. Ankum WM, Mol BW, Van der Veen F, Bossuyt PM (1996) Risk factors for ectopic pregnancy: a meta -analysis: Fertil Steril 65:1093-1099.

8. Anorlu RI, Oluwole A, Abudu OO, Adebajo S, (2005) Risk factors for ectopic pregnancy in Lagos, Nigeria. Acta: Obstet Gynecol Scand 84:184-188.

9. Lewis G (2007) The confidential Enquiry into maternal and child health $(\mathrm{CEMACH})$.Saving Mothers live: reviewing maternal deaths to make mother hood safer 2003-2005.The Seventh Report of Confidential Enquiry in to Maternal Deaths in United Kingdom, London (CEMACH).Edi(Dec) 93-94.

10. The management of tubal pregnancy (2004) Royal College of obstetricians and gynecologists guidelines 21:1-10.

11. Lozean AM, Potter B (2005) Diagnosis and management of ectopic pregnancy. Am Fam Physcian 72: 1707-1714.

12. Bangash N, Ahmed $\mathrm{H}$ (2004) A study of 65 cases of ectopic pregnancy during one year period in military hospital. Pak Armed Forces Med J 54: 205-208.

13. Wasim T (2004) Proportionate morbidity and risk factors of ectopic pregnancy. Ann King Edward Med Coll 10: 298-300.

14. Mahboob U, Mazhar SB (2006) Management of ectopic Pregnancy: A two year study. J Ayub Med Coll Abbottabad 18: 34-37.

15. Khaleeque F, Siddiqui R, JafareyNS (2001) Ectopic pregnancies: a three years study. J Pak Med Assoc 51: 240-242.

16. Ehsan N, Mehmood A (1998) Ectopic pregnancy: An analysis of 62 cases. J Pak Med Assoc 48:26-29.

17. Majhi AK, Roy N, Karmakar KS, Banerjee PK (2007) Ectopic pregnancy-an analysis of 180 cases. J Indian Med Assoc 105:308-312.

18. Shah N, Khan NH (2005) Ectopic Pregnancy: presentation and risk factors. J Coll Physicians Surg Pak 15:535-538.

19. Westrom L (1975) Effect of acute pelvic inflammatory disease on fertiltity. Am J Obstet Gynecol 121:707-713.

20. Aziz S, Wafi BA, Swadi HA (2011) Frequency of ectopic pregnancy in a Medical Centre, Kingdom of Saudia Arabia . J Pak Med Assoc 61: 221-224.

21. Bouyer J, Coste J, Shojaei T, Pouly JL, Fernandez H, et al. (2003) Risk factors for ectopic pregnancy: A comprehensive analysis based on a large case control population based studies in France. Am J Epidemiol; 157:185194.

22. Karaer A, Avsar FA, Batioglu S (2006) Risk factors for ectopic pregnancy: a case control study. Aus NZ J Obstet Gynaecol 46: 521-527.

23. C Tharaux-Deneux, J Bouyer, N Job-Spira et al. (1998) Risk of ectopic pregnancy and previous induced abortion. Am J Public Health 88: 401-405.

24. Cunningham FG (2005) Ectopic pregnancy. In Williams Obstetrics; $22^{\text {nd }}$ ed, New York: Mc Graw -Hill 253-272. 
25. Akaba GO, Agida TE, Onafowokan O (2012) Ectopic pregnancy in Nigeria's federal capital territory: a six year review. Niger J Med 21: 241-245.

26. Gharoro EP, Ifbafe AA (2002) Ectopic pregnancy revisited in Benin City, Nigeria: analysis of 152 cases. Acta Obstet Gynecol Scand 81:1139-1143.

27. Poonam, Upreti D, Banerjee B (2005) Ectopic pregnancy; a two year review from BPKIHS, Nepal. Kathmandu Univ Med J 3: 365-369.

28. Wafaa MF (2008) Diagnosis and management of ectopic pregnancy in King Abdulazia University Hospital: a four year experience. JKAU Med Sci 15:1525.
29. Sy T, Diallo Y, Toure A, Diallo FB, Balde AA, et al. (2009) Management of ectopic pregnancy in Conakry, Guinea. Med Trop 69: 565-568.

30. Shrestha J, Saha R (2012) Comparison of Laparoscopy and Laparotomy in the Surgical Management of ectopic pregnancy. J Coll Physcians Surg Pak 22: 760-764.

31. Najmi RS (1995) Culdocentesis: A useful Diagnostic Tool in ectopic pregnancy. J Coll Physicians Surg Pak 5: 272-274.

32. Tariq TA, KorejoR (1992) Culdocentesis in diagnosis of disturbed ectopic pregnancy. Still a useful procedure in developing countries. J Pak Med Assoc 42: 5-6. 